Research Article

\title{
Self-Triggered Model Predictive Control for Perturbed Underwater Robot Systems
}

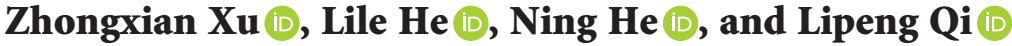 \\ School of Mechanical and Electrical Engineering, Xi'an University of Architecture and Technology, Xi'an 710055, China \\ Correspondence should be addressed to Ning He; hening@xauat.edu.cn
}

Received 9 July 2020; Revised 11 December 2020; Accepted 16 December 2020; Published 4 January 2021

Academic Editor: Ivan Kyrchei

Copyright ( $) 2021$ Zhongxian Xu et al. This is an open access article distributed under the Creative Commons Attribution License, which permits unrestricted use, distribution, and reproduction in any medium, provided the original work is properly cited.

\begin{abstract}
Aiming at solving the control problem of a constrained and perturbed underwater robot, a control method was proposed by combining the self-triggered mechanism and the nonlinear model predictive control (NMPC). The theoretical properties of the kinematic model of the underwater robot, as well as the corresponding MPC controller, are first studied. Then, a novel technique for determining the next update moment of both the optimal control problem and the system state is developed. It is further rigorously proved that the proposed algorithm can (1) stabilize the closed-loop underwater robot system, (2) reduce the time of solving the optimal control problem and (3) save the information transfer resources. Finally, a case study is provided to show the effectiveness of the developed researched scheme.
\end{abstract}

\section{Introduction}

Model predictive control (MPC) has got increasing attention in the past few decades, because of its advantages in dealing with practical control problems [1-4]. One of the main barriers that stops MPC from being applied to more industrial applications is its computational complexity as an optimization problem should be solved at a fixed sampling time repeatedly. The event-based control method can sample according to the relationship between actual state and predicted state to determine the update of the control signal, which is different from its time-driven counterpart, in which the signals are sampled at fixed time intervals, resulting in potential redundant control operations and wasted computation and communication resources. Compared with the traditional sampling mechanism, the event-based sampling scheme is more suitable for MPC as the reduction of sampling instants can significantly save its computation time. In recent years, event-based control mechanism is an important discovery because of its aforementioned advantages; meanwhile, it also presents some design challenges, and one of the most important issues is how to design a triggering condition to achieve a reliable performance $[5,6]$.
Event-based control strategies for a variety of systems have been widely studied. In general, the method mainly includes the event-triggered and self-triggered controls, which are separated by the different triggering mechanism. The former is performed by constantly monitoring the state of the system to determine when control actions must be triggered, while the latter is performed by determining the triggering interval and the next trigger time based on the predicted system states. At present, event-triggered model predictive control strategies are mainly studied for two types of systems: unperturbed systems [7-9] and perturbed systems [10-15]. Similarly, the self-triggered MPC strategies have been investigated in [16-18] and so on. More specifically, in [19], event-triggered and self-triggered controls were introduced in general. The authors mainly highlighted the development history of event-based control and the implementation problems in actual working conditions. For discrete-time linear systems, a robust self-triggered MPC was proposed in [20]. It was emphasized that the role of this MPC scheme is to send the obtained triggering interval to the system at the current triggering moment, before which the system operates in an open-loop mode. In [21], a networked self-triggered MPC scheme was proposed for multi-agent systems which were commonly used in industrial systems, and the problems encountered in the implementation process were 
classified and analyzed. For nonlinear continuous time system with perturbation problem, the event-triggered MPC scheme was studied in [11]. In this scheme, the triggering level was designed to ensure that it will be triggered only under limited conditions. A self-triggered MPC was constructed in [22], in which the next triggering moment was determined by the performance of the pre-designed triggering intervals. In [23], the author proposed a self-triggered strategy in which the design of triggering conditions involves a contraction set which the final state will be finally brought into. In [24], a class of perturbed discrete-time systems was studied. The tube-based random MPC method which required less information from the controlled system was used to deal with the existing constraints to ensure the robust performance of the system under disturbance conditions. In [25], a robust self-triggered MPC algorithm based on the min-max optimization was proposed for discrete-time nonlinear constrained systems with perturbations and model uncertainties. In [26], a self-triggered fuzzy MPC method was proposed for nonlinear robot system with additional constraints to ensure its stable operation. The authors of [27] proposed a robust self-triggered MPC algorithm based on the adaptive control theory for constrained nonlinear discrete-time systems with additive disturbances and achieved feasibility of the optimal control problem and the stability of the closed-loop system. In [28], a new distributed control method based on acceleration gradient method and decoupling strategy was proposed to solve constrained MPC problems for systems composed of nonlinear subsystems. In [15], for nonlinear systems with additional perturbations, an integral-based event-triggered MPC framework was provided to determine the selection of triggering conditions. In [29], the authors proposed an event-based control strategy for the nonlinear distributed systems, which can release the burden of the network communication while achieving the desired global performance. It is worthwhile mentioning that although a lot of interesting approaches as mentioned above have been developed, with the authors' limited knowledge, results on selftriggered MPC of underwater robot systems with bounded disturbances are nearly missing in the literature.

In this paper, a new self-triggered MPC scheme is studied for underwater robot that can be modeled in two reference frame coordinates, the sensors and actuators of which communicate wirelessly with the controller. There are three main novelties and contributions in this paper summarised as follows:

(i) The structural characteristics and mathematical properties of the holonomic kinematic model of the underwater robot are explicitly analyzed, such that the corresponding MPC controller design can be realized in a simple structure to ease its implementation

(ii) A novel self-triggered MPC scheme for the considered underwater robot system with bounded interferences and input constraints is proposed to decrease the computation and communication loads of the robot system (iii) The conditions to guarantee the feasibility of the proposed MPC and the stability of the closed-loop underwater robot system are provided and the resultant theoretical properties are proved

Note that the aforementioned new results (e.g., $[25,27])$ are focusing on combining the min-max optimization or the adaptive control theory with self-triggered MPC to achieve a better theoretical analysis, which, however, will be associated with a relatively larger calculation burden, and may be difficult to be applied in a practical underwater robot due to its short sampling period and limited computing power. In this work, we first analyzed the theoretical properties of the dynamic model of the considered underwater robot system and designed a self-triggered MPC algorithm with a relatively simple structure to facilitate the implementation.

The main motivation of the paper is that the recent researches of self-triggered MPC for underwater robot systems are based on the nonholonomic system model and few results are proposed for the robot characterized by the holonomic system model, which could reflect the dynamic behavior of the robot in a more accurate way. The main difficulty to develop such a control technique is that, given the limited ability of the computation unit of the nowadays underwater robots, the corresponding self-triggered MPC should be associated with a relatively simple structure such that control signal can be efficiently obtained. However, such a simple structure would complicate the design of the conditions to guarantee the feasibility and stability properties from a theoretical point of view. To achieve such an objective, we have first studied the structural characteristics and mathematical properties of the considered holonomic model of the underwater robot systems and obtained a number of useful theoretical results. Based on those results, we have succeeded in designing the self-triggered MPC algorithm in a more standard and simple way and deriving the conditions to ensure the feasibility and stability of the system.

The rest of this work is scheduled as follows. Section 2 models and analyzes the robot system and describes the control problem. The theoretical analysis (feasibility and convergence characteristics of the robot systems) of the NMPC scheme for the considered system is presented in Section 3. Section 4 shows a simulation example to verify the validity of the MPC scheme. Finally, Section 5 gives the conclusions.

\section{Problem Formulation}

2.1. Modeling. The kinematic model of the underwater robot is given as

$$
\dot{x}=f(x, u) \Rightarrow\left[\begin{array}{l}
\dot{\chi} \\
\dot{y} \\
\dot{\gamma}
\end{array}\right]=\left[\begin{array}{ccc}
\cos \varepsilon & -\sin \varepsilon & 0 \\
\sin \varepsilon & \cos \varepsilon & 0 \\
0 & 0 & 1
\end{array}\right]\left[\begin{array}{l}
\mu \\
\nu \\
\omega
\end{array}\right],
$$

and according to matrix multiplication, we further have 


$$
\begin{aligned}
& \dot{\chi}=\mu \cos \varepsilon-\nu \sin \varepsilon, \\
& \dot{y}=\mu \sin \varepsilon+\nu \cos \varepsilon, \\
& \dot{\gamma}=\omega,
\end{aligned}
$$

with $x=[\chi, y, \gamma]^{T}$ denoting the state variable made up of the location $(\chi, y)$ and heading $\gamma$ of the robot under the inertial reference frame. $u=[\mu, \nu, \omega]^{T}$ represents the control inputs vector; meanwhile, $(\mu, \nu)$ is the linear velocity where $\omega$ is angular velocity, shown in the robot reference frame. $R(\varepsilon)$ is the rotation matrix, and it is denoted as

$$
R(\varepsilon)=\left[\begin{array}{ccc}
\cos \varepsilon & -\sin \varepsilon & 0 \\
\sin \varepsilon & \cos \varepsilon & 0 \\
0 & 0 & 1
\end{array}\right]
$$

Considering the actual situation of robot movement, there are the following constraints:

$$
x(t) \in X \subset \mathbb{R}^{3} .
$$

Assuming that the robot's control inputs set is compact, it follows that

$$
u(t) \triangleq[\mu(t), v(t), \omega(t)]^{T} \in U \subset \mathbb{R}^{3} .
$$

The limitations of the control input components are shown as $|\mu| \leq \bar{\mu},|\nu| \leq \bar{\nu}$, and $|\omega| \leq \bar{\omega}$, with $\bar{u}, \bar{\mu}, \bar{\nu}, \bar{\omega} \in \mathbb{R}_{\geq 0}$. Therefore, we deduce $\|u\| \leq \bar{u}$, with $\bar{u}=\sqrt{\bar{\mu}^{2}+\bar{v}^{2}+\bar{\omega}^{2}}$.

Given the model in (1), a perturbed model is shown as follows:

$$
\dot{x}=f(x, u)+\alpha,
$$

with $\alpha(t) \in \mathscr{A} \subset \mathbb{R}^{3}$ representing the disturbance. Suppose the random disturbance is bounded, and we set $\|\alpha(t)\| \leq \bar{\alpha}$.

2.2. NMPC Formulation and Control Design. The desired state can be described as the state vector $x_{d} \triangleq\left[\chi_{d}, y_{d}, \gamma_{d}\right]^{T} \in X$, which can be shown as follows:

$$
\begin{aligned}
& \dot{\chi}_{d}=\mu_{d} \cos \varepsilon_{d}-\nu_{d} \sin \varepsilon_{d}, \\
& \dot{y}_{d}=\mu_{d} \sin \varepsilon_{d}+\nu_{d} \cos \varepsilon_{d}, \\
& \dot{\gamma}_{d}=\omega_{d} .
\end{aligned}
$$

We assume the sequence $\left\{t_{n}\right\}$ is the triggering time, and $u\left(s ; t_{n}\right)$, for $s \in\left[t_{n}, t_{n}+T\right]$, as the control trajectory, where $T$ represents the prediction horizon.

The cost function is formulated as

$$
\begin{aligned}
J\left(\widehat{x}\left(s ; t_{n}\right), \widehat{u}\left(s ; t_{n}\right)\right)= & \int_{t_{n}}^{t_{n}+T} F\left(\widehat{x}\left(s ; t_{n}\right), \widehat{u}\left(s, t_{n}\right)\right) \mathrm{d} s \\
& +E\left(\widehat{x}\left(t_{n}+T\right)\right),
\end{aligned}
$$

subject to

$$
\begin{aligned}
\dot{\hat{x}} & =f\left(\widehat{x}\left(s ; t_{n}\right), \widehat{u}\left(s ; t_{n}\right)\right), \quad s \in\left[t_{n}, t_{n}+T\right], \\
\widehat{u}\left(s ; t_{n}\right) & \in U, \quad s \in\left[t_{n}, t_{n}+T\right], \\
\widehat{x}\left(s ; t_{n}\right) & \in X_{t-t_{n}}, \quad s \in\left[t_{n}, t_{n}+T\right], \\
\widehat{x}\left(t_{n}+T\right) & \in \xi_{f},
\end{aligned}
$$

where $\widehat{u}(t)$ is the predicted control sequence and $\widehat{x}(t)$ is the predicted state sequence of the robot system, which satisfies nominal system (1). $F$ is the running cost function while $E$ is terminal cost function, and they can be represented by $F(x, u)=x^{T} Q x+u^{T} R u$ and $E(x, u)=x^{T} P x$, respectively. We set the weighting matrices as $Q=\operatorname{diag}\left\{q_{1}, q_{2}, q_{3}\right\}$, $R=\operatorname{diag}\left\{r_{1}, r_{2}\right\}$, and $P=\operatorname{diag}\left\{p_{1}, p_{2}, p_{3}\right\}$, where $Q, R$, and $P$ are diagonal matrices. For further analysis, we define that $\widehat{x}\left(s ; t_{n}\right)$ means the predicted state at time $s$ with $s \in\left[t_{n}, t_{n}+\right.$ $T]$ of the nominal system.

2.3. Problem Statement. In order to prove that the NMPC scheme can generate controllers guaranteeing the robust stability, the following stability assumptions are proposed.

Assumption 1. Assume that $\xi \subset X$ is a minimum robust positive invariant set of nominal system (1), and $\xi \triangleq\left\{x \in X:\|x\| \leq \delta_{0}\right\}$, with $\delta_{0}>0$.

Assumption 2. Assume that there exists a local stabilizing controller, satisfying $(\partial E / \partial x) f\left(x(s), u_{T}(x(s))\right) \leq-F(x(s)$, $\left.u_{T}(x(s))\right), \forall x \in \xi$.

Assumption 3. $E(x)=x^{T} P x \leq \rho_{\xi}$, for the set $\xi$, where $\rho_{\xi}=\max \left\{p_{1}, p_{2}, p_{3}\right\} \delta_{0}^{2}>0$. We further suppose that $\xi=\left\{x \in X: u^{T}(x) \in U\right\}$. Take $\rho_{\xi_{f}} \in\left(0, \rho_{\xi}\right)$ and assume that $\xi_{f}=\left\{x \in \mathbb{R}^{3}: E(x) \leq \rho_{\xi_{f}}\right\}, \forall x \in \xi, f\left(x, u_{T}\right) \in \xi_{f}$.

At time $t_{n}$, the optimal control trajectory $u^{*}\left(s ; t_{n}\right)$ is obtained by the solution of (9), for $s \in\left[t_{n}, t_{n}+T\right]$. We get

$$
u(s)=u^{*}\left(s ; t_{n}\right), \quad s \in\left[t_{n}, t_{n+1}\right) .
$$

The control law should be used to the plant in the form of an open-loop manner within the time intervals $\left[t_{n}, t_{n+1}\right)$. The meaningful thing is how long this time interval is. Later in this article, a new self-triggered MPC scheme will be studied to save the time to solve the problem. More importantly, the framework proposed later can be used to calculate not only the control law, but also the time of the next triggering moment $t_{n+1}$.

Considering that system (6) is affected by constraints (4) and (5), our goal is to (i) design a self-triggered state feedback robust control approach through (9), so that the state of system (6) converges to an invariant set, and (ii) design a triggering strategy to determine the triggering interval and the next control update time.

First, some important performances of the nominal system (1) are proved in the following lemmas. 
Lemma 1. The nominal underwater robot system (1) is Lipschitz continuous, with Lipschitz constant Proof. Considering the Euclidean norm, we get $L_{f} \triangleq \sqrt{2\left(\mu^{2}+v^{2}\right)}$, in the state $x$.

$$
\begin{aligned}
\left\|f\left(x_{1}, u\right)-f\left(x_{2}, u\right)\right\|^{2} & =\| \begin{array}{c}
\mu \cos \epsilon_{1}-\nu \sin \epsilon_{1}-\mu \cos \epsilon_{2}+\nu \sin \epsilon_{2} \\
\mu \sin \epsilon_{1}+\nu \cos \epsilon_{1}-\mu \sin \epsilon_{2}-\nu \cos \epsilon_{2} \\
\omega_{1}-\omega_{2}
\end{array}
\end{aligned} \|^{2}
$$

It means that $\left\|f\left(x_{1}, u\right)-f\left(x_{2}, u\right)\right\| \leq \sqrt{2\left(\mu^{2}+v^{2}\right)} \| x_{1}-$ $x_{2} \|$ for all $x_{1}, x_{2} \in X$.

Lemma 2. For the disturbed underwater robot system, the actual state $x\left(s ; t_{n}\right)$ and the predicted state $\hat{x}\left(s ; t_{n}\right)$ are inconsistent, with the deviation being

$$
\left\|x\left(s ; t_{n}\right)-\widehat{x}\left(s ; t_{n}\right)\right\| \leq \beta(s),
$$

with $\beta(s) \triangleq\left(2 \sqrt{2\left(\bar{\mu}^{2}+\bar{\nu}\right)^{2}}+\bar{\omega}\right) s, \forall s \in\left[t_{n}, t_{n}+T\right]$.

Proof. For $s \in\left[t_{n}, t_{n+1}\right]$, we get

$$
\begin{aligned}
& \left\|x\left(s ; t_{n}\right)-\widehat{x}\left(s ; t_{n}\right)\right\| \\
& =\left\|x\left(t_{n}\right)+\int_{t_{n}}^{t_{n}+t} f(x(s), u(s)) \mathrm{d} s+\int_{t_{n}}^{t_{n}+t} \omega(s) \mathrm{d} s-\widehat{x}\left(t_{n}\right)-\int_{t_{n}}^{t_{n}+t} f(\widehat{x}(s), \widehat{u}(s)) \mathrm{d} s\right\| \\
& \leq\left\|\int_{t_{n}}^{t_{n}+t}[f(x(s), u(s))-f(\widehat{x}(s), \widehat{u}(s))] \mathrm{d} s\right\|+\int_{t_{n}}^{t_{n}+t} \omega(s) \mathrm{d} s \leq\left(2 \sqrt{2\left(\bar{\mu}^{2}+\bar{v}\right)^{2}}+\bar{\omega}\right) t .
\end{aligned}
$$

Because of the disturbance, the constraint set of the system state needs to be modified to ensure the robustness of the system. Therefore, we consider a restricted constraint set $X_{t-t_{n}}$, with $X_{t-t_{n}} \subset X$. Based on the constraint tightening technique, when the control trajectory of (9) is applied to system (6), the resulting states conform to the state constraint set $X$. To sum up, the restricted constraint set is tightened to $X_{t-t_{n}}=X \sim S_{t-t_{n}}$, where $S_{t-t_{n}}=\left\{x \in \mathbb{R}^{3}:\|x\| \leq \beta\right.$ $\left.\left(t-t_{n}\right)\right\}$, with $t \in\left[t_{n}, t_{n}+T\right]$, and the notation “ $\sim$ " means the Pontryagin difference. In the following, we further show that the running cost of the MPC is also Lipschitz.

Lemma 3. The running cost $F(x, u)$ is Lipschitz with the Lipschitz constant $L_{F} \triangleq 2\left(L_{\text {max }}^{2}+(\pi / 2)^{2}\right)^{1 / 2} \lambda_{\text {max }}(Q)$ in the state $x$, where $\lambda_{\max }(Q)$ is the maximum eigenvalue of the matrix $Q$, and $L_{\max }$ is the maximum distance between the robot's current position and the target.

Proof. Given the definition of the MPC cost function, we have $\quad\left\|F\left(x_{1}, u\right)-F\left(x_{2}, u\right)\right\|=\left\|x_{1}^{T} Q x_{1}-x_{2}^{T} Q x_{2}\right\| \leq\left(\left\|x_{1}\right\|-\right.$ $\left.\left\|x_{2}\right\|\right) \lambda_{\max }(Q)\left\|x_{1}-x_{2}\right\|$.

Notice that since $\forall x \in X$, and we know $\|x\|^{2} \leq\|\chi\|^{2}+\|y\|^{2}+\|\epsilon\|^{2} \leq L_{\max }^{2}+(\pi / 2)^{2}$, we can derive that $L_{F} \triangleq 2\left(L_{\text {max }}^{2}+(\pi / 2)^{2}\right)^{1 / 2} \lambda_{\text {max }}(Q)$.

\section{Theoretical Properties Analysis}

The stability analysis of the system will be carried out in this section. Since the system is perturbed by disturbances, we can only ensure the result of its ultimate boundedness. The theoretical analysis of the considered system under the proposed predictive controller regarding the feasibility and the convergence of the closed-loop system are proposed in the following.

The dual-mode control scheme is adopted in this work. In other words, once the state belongs to the terminal set, we will apply the state local control law $u(t)=K x(t)$, instead of solving the optimal control problem, thus saving computation and communication resources. The self-triggered MPC algorithm we proposed is shown in Algorithm 1 as follows.

3.1. Feasibility Property Analysis. The feasibility property of the considered system is shown in the following.

Theorem 1. Suppose that Assumptions 1-3 hold. If T meets the condition $T \leq\left(\rho_{\xi}-\rho_{\xi_{f}} / L_{E}\left(2 \sqrt{2\left(\bar{\mu}^{2}+\bar{\nu}\right)^{2}}+\bar{\omega}\right)\right)$, then the proposed MPC scheme is feasible. 
(1) while $x\left(s ; t_{n}\right) \notin \xi_{f}$ do

(2) Solve optimization problem;

(3) while $t_{n+1}$ is not triggered do

(4) Apply the input $u^{*}\left(s ; t_{n}\right)$;

(5) end while

(6) Compute the next triggering time $t_{n+1}$;

(7) $n=n+1$;

(8) end while

(9) Apply the state feedback control law.

Algorithm 1: Self-triggered MPC.

Proof. We construct $\widetilde{u}\left(s ; t_{n+1}\right)$ as a common feasible control input, at $t_{n+1}$, as follows:

$$
\widetilde{u}\left(s ; t_{n+1}\right)= \begin{cases}\widehat{u}^{*}\left(s ; t_{n}\right), & s \in\left[t_{n+1}, t_{n}+T\right], \\ K \widehat{x}^{*}\left(s ; t_{n}\right), & s \in\left[t_{n}+T, t_{n+1}+T\right],\end{cases}
$$

where $K \hat{x}^{*}\left(s ; t_{n}\right)$ is the local feedback control law. Given the considered control signal, we have $E\left(\hat{x}\left(t_{n}+T ; t_{n+1}\right)\right.$ $\leq E\left(\widehat{x}\left(t_{n}+T ; t_{n}\right)\right)+L_{E} \beta(T) \leq \rho_{\xi_{f}}+L_{E}$

$\left(2 \sqrt{2\left(\bar{\mu}^{2}+\bar{\nu}\right)^{2}}+\bar{\omega}\right) T \leq \rho_{\xi}$. Note that the inequality $2 \sqrt{2\left(\bar{\mu}^{2}+\bar{\nu}\right)^{2}}+\bar{\omega} \leq\left(\rho_{\xi}-\rho_{\xi_{f}} / L_{E} T\right)$ can guarantee that the uncertainty is limited. Considering that $\left\|x\left(s ; t_{n}\right)-\widehat{x}\left(s ; t_{n}\right)\right\| \leq \beta(t)$, for all $s \in\left[t_{n}, t_{n}+T\right]$, we have $\widehat{x}\left(s ; t_{n}\right) \in X_{t-t_{n}}$, and then $\hat{x}\left(s ; t_{n+1}\right) \in X_{t-t_{n}}$ can be verified. Furthermore, it can be shown that $\widetilde{u}\left(s ; t_{n+1}\right) \in U$ for all $s \in\left[t_{n+1}, t_{n+1}+T\right]$. Then, the proof is completed.
3.2. Convergence Property Analysis. The convergence property analysis of the considered system is shown in the following.

Theorem 2. Consider system (6) with constraints (4) and (5) and suppose Assumptions 1-3 are valid. The control process is shown as Algorithm 1. The state will shrink to a compact set $\xi_{f}$ in limited time. Finally, the closed-loop system remains stable.

Proof. We choose the optimal cost $J^{*}\left(u^{*}\left(s ; t_{n}\right)\right.$, $\left.x\left(t_{n}\right)\right) \triangleq J^{*}\left(t_{n}\right)$ as the Lyapunov function. Then, the cost of the feasible trajectory is denoted as $\bar{J}^{*}\left(\tilde{u}\left(s ; t_{n+1}\right) t, n x q\left(t_{n+1}\right)\right) \triangleq \bar{J}\left(t_{n+1}\right)$, and $t_{n}, t_{n+1}$ are consecutive triggering moments. Considering the predicted state $\bar{x}\left(s ; t_{n+1}\right)$, with $s \geq t_{n+1}$, according to the actual state at time $t_{n+1}, \bar{u}(s ; x(n+1))$ indicates the feasible control trajectory from (14).

Set $x_{1}(s)=\bar{x}\left(s ; t_{n+1}\right), u_{1}(s)=\bar{u}\left(s ; t_{n+1}\right), x_{2}(s)=\widehat{x}\left(s ; t_{n}\right)$ and $u_{2}(s)=u^{*}\left(s ; t_{n}\right)$.

According to the above definition, the difference between the feasible and optimal costs is

$$
\begin{aligned}
\bar{J}\left(t_{n+1}\right)-J^{*}\left(t_{n}\right)= & \int_{t_{n+1}}^{t_{n}+T} F\left(x_{1}(s), u_{1}(s)\right) \mathrm{d} s+E\left(x_{1}\left(t_{n+1}+T\right)\right) \\
& +\int_{t_{n}+T}^{t_{n+1}+T} F\left(x_{1}(s), u_{1}(s)\right) \mathrm{d} s-\int_{t_{n}}^{t_{n+1}} F\left(x_{2}(s), u_{2}(s)\right) \mathrm{d} s \\
& -\int_{t_{n+1}}^{t_{n}+T} F\left(x_{2}(s), u_{2}(s)\right) \mathrm{d} s-E\left(x_{2}\left(t_{n}+T\right)\right) .
\end{aligned}
$$

From (14), we know that $u_{1}(t) \equiv u_{2}(t) \equiv \bar{u}(t)$ for $t \in\left[t_{n+1}, t_{n}+T\right]$. Applying this to system (1), we can get

$$
\left\|x\left(t_{n+1}\right)-\widehat{x}\left(t_{n+1} ; t_{n}\right)\right\| \leq \beta\left(t_{n+1}-t_{n}\right) .
$$

With the help of (16), the difference between the running costs becomes

$$
\begin{aligned}
& \int_{t_{n+1}}^{t_{n+1}+T} F\left(x_{1}(s), u_{1}(s)\right) \mathrm{d} s-\int_{t_{n+1}}^{t_{n+1}+T} F\left(x_{2}(s), u_{2}(s)\right) \mathrm{d} s \\
& \quad \leq L_{F} \int_{t_{n+1}}^{t_{n+1}+T}\left\|x_{1}(s)-x_{2}(s)\right\| \mathrm{d} s=L_{F}\left(2 \sqrt{2\left(\bar{\mu}^{2}+\bar{\nu}\right)^{2}}+\bar{\omega}\right)\left(t_{n+1}-t_{n}\right)\left(t_{n}+T-t_{n+1}\right) \geq 0 .
\end{aligned}
$$


Considering the property from Assumption 2 for $s \in\left[t_{n}+T, t_{n+1}+T\right]$, we arrive at the following relationship:

$$
\begin{aligned}
& E\left(x_{1}\left(t_{n+1}+T\right)\right)+\int_{t_{n}+T}^{t_{n+1}+T} F\left(x_{1}(s), u_{1}(s)\right) \mathrm{d} s-E\left(x_{2}\left(t_{n}+T\right)\right)-E\left(x_{1}\left(t_{n}+T\right)\right)+E\left(x_{1}\left(t_{n}+T\right)\right) \\
& \quad \leq L_{E}\left(2 \sqrt{2\left(\bar{\mu}^{2}+\bar{\nu}\right)^{2}}+\bar{\omega}\right)\left(t_{n+1}-t_{n}\right) \geq 0 .
\end{aligned}
$$

As the function is positive definite, we can draw the conclusion that

$$
\int_{t_{n}}^{t_{n+1}} F\left(x_{2}(s), u_{2}(s)\right) \mathrm{d} s \geq L_{Q}\left(t_{n+1}\right) \geq 0
$$

with $L_{Q}(t) \triangleq \min \left\{q_{1}, q_{2}, q_{3}, r_{1}, r_{2}\right\} \cdot \int_{t}^{t}\left\|\widehat{x}\left(s ; t_{n}\right)\right\|^{2} \mathrm{~d} s \quad$ for $t>t_{n}$. So, if we substitute (17), (18), and (19) into (15), we get

$$
\bar{J}\left(t_{n+1}\right)-J^{*}\left(t_{n}\right)=L_{F}\left(2 \sqrt{2\left(\bar{\mu}^{2}+\bar{\nu}\right)^{2}}+\bar{\omega}\right)\left(t_{n+1}-t_{n}\right)\left(t_{n}+T-t_{n+1}\right)+L_{E}\left(2 \sqrt{2\left(\bar{\mu}^{2}+\bar{\nu}\right)^{2}}+\bar{\omega}\right)\left(t_{n+1}-t_{n}\right)-L_{Q}\left(t_{n+1}\right)
$$

Then, we have

$$
J^{*}\left(t_{n+1}\right)-J^{*}\left(t_{n}\right) \leq \bar{J}\left(t_{n+1}\right)-J^{*}\left(t_{n}\right) .
$$

Now, the Lyapunov function $J^{*}(\cdot)$ has been certified to be decreasing, and it has turned out that the closed-loop system state ends up in a compact set $\xi_{f}$. The proof is completed.

3.3. Discussion on the Self-Triggered System. Denote $t_{n}$ as a triggering instant, and $t_{n}, t_{n+1}$ as two consecutive triggering moments; the next control update instant $t_{n+1}$ can then be found. More specifically, if we have time $t$ satisfying $t \in\left[t_{n}, t_{n+1}\right]$, considering (20) and (21), we get

$$
\begin{aligned}
J^{*}(t)-J^{*}\left(t_{n}\right) \leq & L_{F}\left(2 \sqrt{2\left(\bar{\mu}^{2}+\bar{\nu}\right)^{2}}+\bar{\omega}\right)\left(t-t_{n}\right)\left(t_{n}+T-t\right) \\
& +L_{E}\left(2 \sqrt{2\left(\bar{\mu}^{2}+\bar{\nu}\right)^{2}}+\bar{\omega}\right)\left(t-t_{n}\right)-L_{Q}(t) .
\end{aligned}
$$

Further, we can get

$$
\begin{aligned}
& L_{F}\left(2 \sqrt{2\left(\bar{\mu}^{2}+\bar{\nu}\right)^{2}}+\bar{\omega}\right)\left(t-t_{n}\right)\left(t_{n}+T-t\right) \\
& \quad+L_{E}\left(2 \sqrt{2\left(\bar{\mu}^{2}+\bar{\nu}\right)^{2}}+\bar{\omega}\right)\left(t-t_{n}\right) \leq \Psi L_{Q}(t),
\end{aligned}
$$

with $0<\Psi<1$. Together with (22), we have

$$
J^{*}(t)-J^{*}\left(t_{n}\right) \leq(\Psi-1) L_{Q}(t)
$$

This means that convergence property is still guaranteed if we can prove $\Psi<1$. Therefore, when time $t$ does not meet (24), the system should be triggered again.

At time $t_{n}$, the control signal is updated, and meanwhile, future output signals for $\left[t_{n}, t_{n}+T\right]$ are provided. We can get

$$
\begin{aligned}
& \left(2 \sqrt{2\left(\bar{\mu}^{2}+\bar{\nu}\right)^{2}}+\bar{\omega}\right)\left[L_{F}\left(t_{n}+T-t\right)+L_{E}\right]\left(t-t_{n}\right) \\
& =\Psi L_{Q}(t) .
\end{aligned}
$$

Solve equation (23) and we can get the next update time $t_{n+1}$, and $u(s)=u^{*}\left(s ; t_{n}\right)$ is used to the system in the form of an open-loop manner within $s \in\left[t_{n}, t_{n+1}\right)$. In the next step, the state calculated at the time of update is taken at time $t_{n+1}$. The MPC controller cycles through the process from the beginning until the closed-loop system state ends up in a compact set $\xi_{f}$.

\section{Simulation Results}

Simulation and comparison results of the proposed selftriggered MPC strategy are provided for the considered underwater robot system. Consider system (6) with constraints (4) and (5) and suppose Assumptions 1-3 hold, the disturbance is bounded by $\|\alpha\| \leq 0.5$, and the input constraint is set as $\|u(t)\| \leq 2$. When the simulation starts, the state of the robot is $x_{0}=[-45,15,-\pi / 6]^{T}$, and the desired position is $x_{d}=[0,0,0]^{T}$. The goal is to decrease the frequency of control signal updates and the time of solving the optimal control problem without significantly affecting control performance.

The simulation is carried out according to the selftriggered MPC scheme in Algorithm 1. Figure 1 shows the 


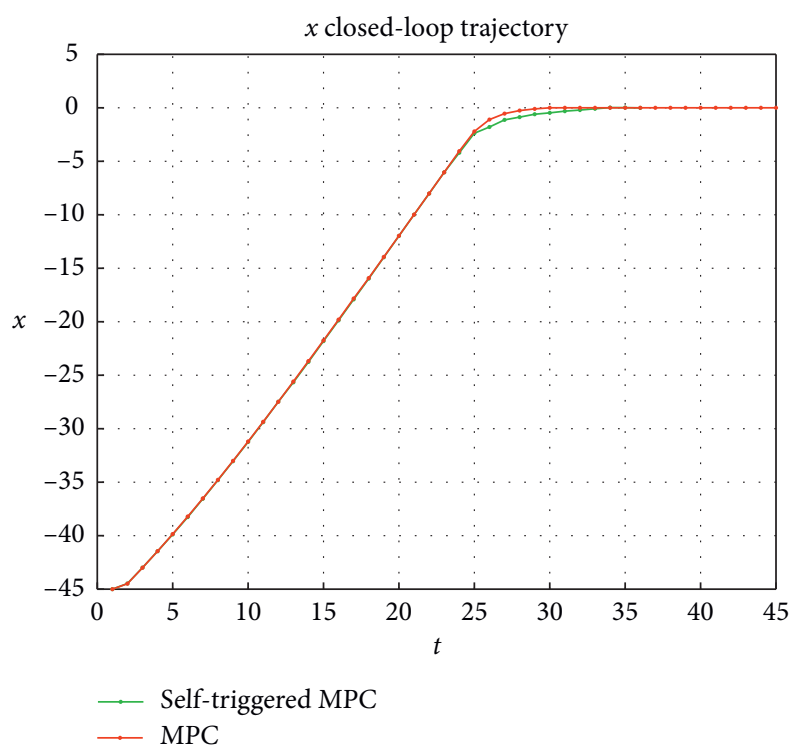

Figure 1: Comparison of the trajectories of $\chi$ obtained via the selfand time-triggered methods.

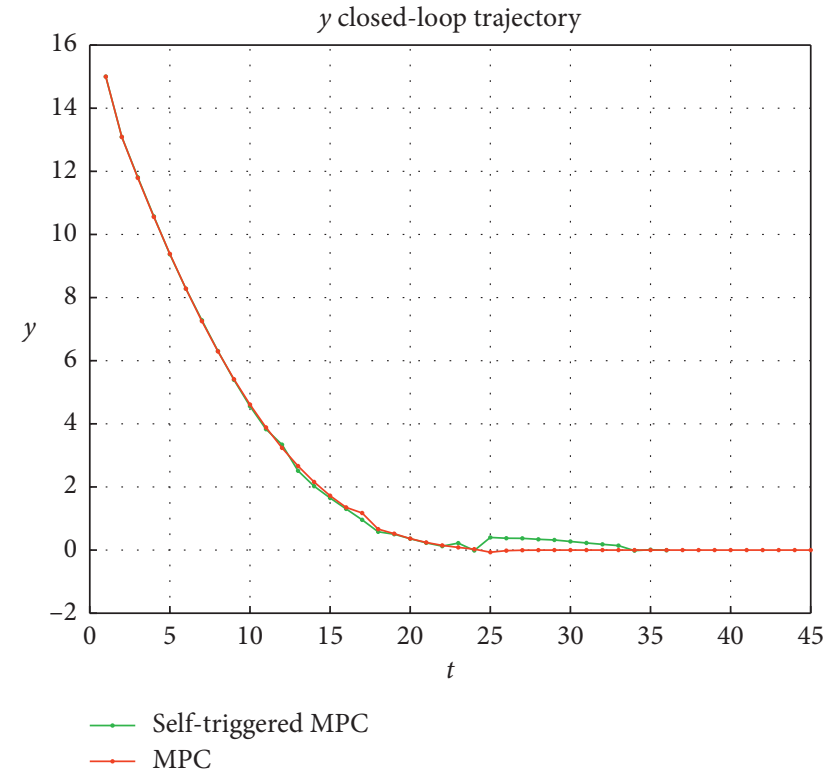

FIGURE 2: Comparison of the trajectories of $y$ obtained via the selfand time-triggered methods.

state trajectories $\chi$ obtained by the self-triggered MPC and its time-triggered counterpart, which are represented by a green line and a red line, respectively. It can be seen from the figure that the state trajectories under the two control methods are almost completely identical, and there is only a small difference between the states while they are approaching the stable value. Figure 2 shows the difference between the state trajectories $y$, and similar conclusions can be drawn as above. In order to show the comparison more clearly, the robot motion trajectories obtained by different methods in a two-dimensional plane (including the coordinates and orientation of the robot) are shown in Figure 3. Finally,

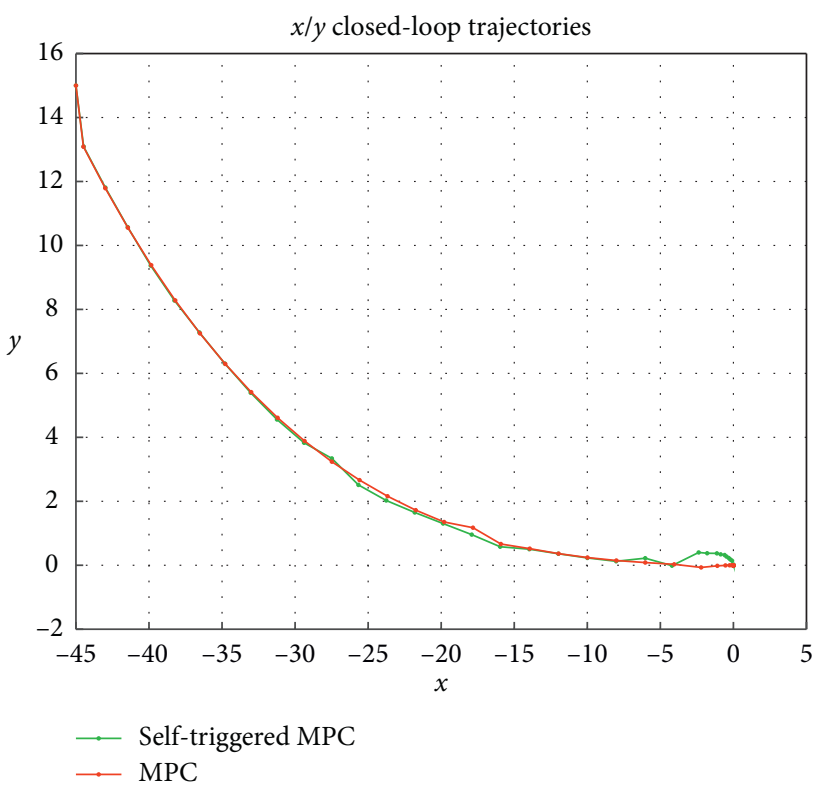

FIGURE 3: Comparison of state trajectories of the underwater robot controlled via the self- and time-triggered model predictive control methods.

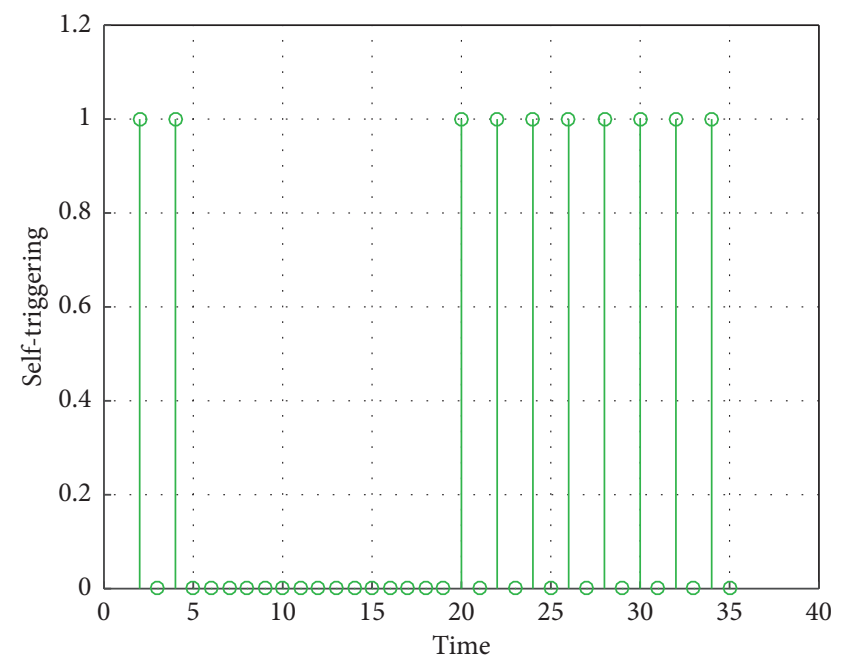

FIGURE 4: The triggering time instants of the proposed method.

Figure 4 shows the triggering moments of the self-triggered MPC scheme designed in this work. If the vertical axis is 1 , the MPC was triggered. If it is 0 , the MPC was not triggered. The time-triggered framework is not shown in the figure because it is periodically triggered at each sampling instant. It is noted that the self-triggered MPC is only triggered 11 times out of 45 times over the whole simulation time, which means that computational load of the system is reduced by $76 \%$ without significantly reducing the control performance.

\section{Conclusions}

In this study, a self-triggered model predictive control scheme has been designed for the underwater robot systems 
with constraints and perturbations. This scheme is used to determine the moment when the next trigger will occur, and its purpose is to maximize the sampling interval. By taking the cost function as the Lyapunov function, the triggering condition is obtained through the stability calculation. The dual-mode control scheme is applied to further decrease the triggering frequency and to save signal transmission resources. To guarantee the feasibility of the strategy and the stability of the system, the strict theoretical analyses and proofs are given in detail. The validity of the theoretical results is also verified by a simulation example.

\section{Data Availability}

The main contribution of this paper is the theoretical derivation and proofs, and data are not the main material supporting the contribution.

\section{Conflicts of Interest}

The authors declare that they have no conflicts of interest.

\section{Acknowledgments}

This work was supported by National Natural Science Foundation of China (Grant no. 61903291) and Basic Research Plan in Shaanxi Province of China (Grant no. 2019JQ-004).

\section{References}

[1] H. Chen and F. Allgöwer, "A quasi-infinite horizon nonlinear model predictive control scheme with guaranteed stability," Automatica, vol. 34, no. 10, pp. 1205-1217, 1998.

[2] P. Varutti, B. Kern, T. Faulwasser, and R. Findeisen, "Eventbased model predictive control for networked control systems," in Proceedings of the 48h IEEE Conference on Decision and Control (CDC) Held Jointly with 2009 28th Chinese Control Conference, pp. 567-572, IEEE, Shanghai, China, December 2009.

[3] A. Anta and P. Tabuada, "To sample or not to sample: selftriggered control for nonlinear systems," IEEE Transactions on Automatic Control, vol. 55, no. 9, pp. 2030-2042, 2010.

[4] A. Eqtami, D. V. Dimarogonas, and K. J. Kyriakopoulos, "Event-triggered control for discrete-time systems," in Proceedings of the 2010 American Control Conference, pp. 47194724, IEEE, Baltimore, MD, USA, July 2010.

[5] E. Henriksson, D. E. Quevedo, H. Sandberg, and K. H. Johansson, "Self-triggered model predictive control for network scheduling and control," IFAC Proceedings Volumes, vol. 45, no. 15, pp. 432-438, 2012.

[6] W. H. Heemels, M. Donkers, and A. R. Teel, "Periodic eventtriggered control for linear systems," IEEE Transactions on Automatic Control, vol. 58, no. 4, pp. 847-861, 2012.

[7] E. Garcia and P. J. Antsaklis, "Model-based event-triggered control with time-varying network delays," in Proceedings of the 2011 50th IEEE Conference on Decision and Control and European Control Conference, pp. 1650-1655, IEEE, Orlando, FL, USA, December 2011.

[8] X. Mi, Y. Zou, and S. Li, "Event-triggered MPC design for distributed systems toward global performance,"
International Journal of Robust and Nonlinear Control, vol. 28, no. 4, pp. 1474-1495, 2018.

[9] M. Bingxian, Z. Kunwu, X. Feng, and S. Yang, "Event-based rendezvous control for a group of robots with asynchronous periodic detection and communication time delays," IEEE Transactions on Cybernetics, vol. 49, no. 7, pp. 1-10, 2018.

[10] D. Lehmann, E. Henriksson, and K. H. Johansson, "Eventtriggered model predictive control of discrete-time linear systems subject to disturbances," in Proceedings of the 2013 European Control Conference (ECC), pp. 1156-1161, IEEE, Zurich, Switzerland, July 2013.

[11] H. Li and Y. Shi, "Event-triggered robust model predictive control of continuous-time nonlinear systems," Automatica, vol. 50, no. 5, pp. 1507-1513, 2014.

[12] D. Shi, T. Chen, and L. Shi, "An event-triggered approach to state estimation with multiple point- and set-valued measurements," Automatica, vol. 50, no. 6, pp. 1641-1648, 2014.

[13] Z. Sun, L. Dai, Y. Xia, and K. Liu, "Event-based model predictive tracking control of nonholonomic systems with coupled input constraint and bounded disturbances," IEEE Transactions on Automatic Control, vol. 63, no. 2, pp. 608-615, 2017.

[14] C. Liu, J. Gao, H. Li, and D. Xu, "Aperiodic robust model predictive control for constrained continuous-time nonlinear systems: an event-triggered approach," IEEE Transactions on Cybernetics, vol. 48, no. 5, pp. 1397-1405, 2017.

[15] Q. Sun, J. Chen, and Y. Shi, "Integral-type event-triggered model predictive control of nonlinear systems with additive disturbance," IEEE Transactions on Cybernetics, In press.

[16] A. M. Memon and M. S. Mahmoud, "Evaluation of novel selftriggering method for optimisation of communication and control," IET Control Theory \& Applications, vol. 10, no. 1, pp. 76-83, 2016.

[17] H. Yu and F. Hao, "Input-to-state stability of integral-based event-triggered control for linear plants," Automatica, vol. 85, pp. 248-255, 2017.

[18] L. Dai, Y. Gao, L. Xie, K. H. Johansson, and Y. Xia, "Stochastic self-triggered model predictive control for linear systems with probabilistic constraints," Automatica, vol. 92, pp. 9-17, 2018.

[19] W. Heemels, K. H. Johansson, and P. Tabuada, "An introduction to event-triggered and self-triggered control," in Proceedings of the 2012 IEEE 51st IEEE Conference on Decision and Control (CDC), pp. 3270-3285, IEEE, Maui, HI, USA, December 2012.

[20] J. D. J. B. Berglind, T. M. P. Gommans, and W. P. M. H. Heemels, "Self-triggered MPC for constrained linear systems and quadratic costs," IFAC Proceedings Volumes, vol. 45, no. 17, pp. 342-348, 2012.

[21] A. Eqtami, S. Heshmati-Alamdari, D. V. Dimarogonas, and K. J. Kyriakopoulos, "Self-triggered model predictive control for nonholonomic systems," in Proceedings of the $2013 \mathrm{Eu}$ ropean Control Conference (ECC), Zurich, Switzerland, July 2013.

[22] T. M. P. Gommans and W. P. M. H. Heemels, "Resourceaware MPC for constrained nonlinear systems: a self-triggered control approach," Systems \& Control Letters, vol. 79, pp. 59-67, 2015.

[23] K. Hashimoto, S. Adachi, and D. V. Dimarogonas, "Selftriggered control for constrained systems: a contractive setbased approach," in Proceedings of the 2017 American Control Conference (ACC), pp. 1011-1016, IEEE, Seattle, WA, USA, May 2017.

[24] F. D. Brunner, W. P. M. H. Heemels, and F. Allgöwer, "Robust event-triggered MPC with guaranteed asymptotic bound and 
average sampling rate," IEEE Transactions on Automatic Control, vol. 62, no. 11, pp. 5694-5709, 2017.

[25] C. Liu, H. Li, J. Gao, and D. Xu, "Robust self-triggered minmax model predictive control for discrete-time nonlinear systems," Automatica, vol. 89, pp. 333-339, 2018.

[26] Y. Su, Q. Wang, and C. Sun, "Self-triggered robust model predictive control for nonlinear systems with bounded disturbances," IET Control Theory \& Applications, vol. 13, no. 9, pp. 1336-1343, 2018.

[27] Z. Sun, L. Dai, K. Liu, D. V. Dimarogonas, and Y. Xia, "Robust self-triggered MPC with adaptive prediction horizon for perturbed nonlinear systems," IEEE Transactions on Automatic Control, vol. 64, no. 11, pp. 4780-4787, 2019.

[28] D. E. H. Hammami, S. Maraoui, and K. Bouzrara, "Distributed MPC control with dual decomposition and accelerated gradient methods: application for wind-solar systems," in Proceedings of the 2019 International Conference on Signal, Control and Communication (SCC), pp. 63-68, IEEE, Hammamet, Tunisia, December 2019.

[29] D. E. H. Hammami, S. Maraoui, and K. Bouzrara, "Nonlinear distributed model predictive control with dual decomposition and event-based communication approach," Transactions of the Institute of Measurement and Control, vol. 42, no. 15, pp. 2929-2940, 2020. 